\title{
Constraint-free theories of gravitation
}

\author{
Frank B Estabrook, R Steve Robinson and Hugo D Wahlquist \\ Jet Propulsion Laboratory 169-327 \\ California Institute of Technology 4800 Oak Grove Drive \\ Pasadena, California, 91109, USA
}

August 3, 1998

\begin{abstract}
Lovelock actions (more precisely, extended Gauss-Bonnet forms) when varied as Cartan forms on subspaces of higher dimensional flat Riemannian manifolds, generate well set, causal exterior differential systems. In particular, the Einstein-Hilbert action 4-form, varied on a 4 dimensional subspace of $E_{10}$, yields a well set generalized theory of gravity having no constraints. Ricci-flat solutions are selected by initial conditions on a bounding 3-space.
\end{abstract}

\section{Introduction}

Field theoretic Lagrangians (involving, say, $p$ independent variables and $n-p$ dependent variables) can be systematically "extended" to derive so-called Cartan forms, $\boldsymbol{p}$-forms that are analogs of the Cartan Lagrangian 1 -forms for variational principles in the differential geometric formulation of classical mechanics [1] [2] [3] [4] [5] [6] [7] [8] [9] [10] [11] [12] [13] [14]. Conversely, Cartan forms of degree $p$ can often be postulated, and field theories in $p$ dimensions can be obtained from them by variation, using Cartan's formalism of exterior differential systems (EDS). Such Cartan forms and the corresponding variational EDS live in a "target" space that is a suitable bundle over the $n$ dimensional space of all independent and dependent variables. The target space may be a jet bundle over $p, n-p$ variables, but other targets occur in contexts such as geometry or string theory.

The sort of variational EDS we discuss in the following is generated by structure 1 -forms and their closure 2 -forms expressing the immersion of $p$-dimensions, 
or a subbundle over it, into the target bundle, and by a set of $p$-forms expressing the field equations or equivalent geometric conditions. To justify use of a proposed Cartan form it must be shown to lead, by arbitrary variation, to such $p$-form field equations. The derived EDS must be closed (thereby including all integrability conditions and being self-consistent), well set, and, in fact, causal, i.e., it must satisfy Cartan's criteria for the so-called characteristic integers $s_{i}$ of the EDS, and have $s_{p}=0$ [7] [15] [16] [17]. This is tantamount to the satisfaction of a Cauchy-Kowalewski existence and uniqueness theorem for the implied set of partial differential equations.

An EDS derived variationally in this way is constraint-free, since it is generated by no forms of degree less than $p$, other than those expressing the immersion. An example from string theory is given in Section 2. Another example is vacuum electrodynamics, formulated on the jet bundle $J^{1}(4,4)$ over a four-potential $A_{i}$ [9]. Constraint-free dynamics can perhaps be adapted to efficient techniques for numerical solution, with initial conditions set on $p-1$ dimensional surfaces immersed in the target space. Quantized versions may also be more straightforward to formulate, as the example from electrodynamics shows.

Extremals of a $p$-form Lagrangian set with the frame coordinates of a larger $n$ dimensional immersing space as a target can be different - can satisfy different dynamics and partial differential equations - from those derived from the same Lagrangian intrinsically, that is to say set on a target that is a jet bundle over $p$ independent variables [18]. These target spaces have intrinsic basis forms that satisfy different structure or contact equations. The classic Plateau soap bubble and simple strings (the example in Section 2) are prototypes of Lagrangians set on immersing orthonormal frame bundles, targets quite different from jet bundles.

We have calculated sets of Cartan characteristic integers for EDS derived from Cartan forms expressing several of the so-called Lovelock Lagrangians [19]. Also known as dimensionally extended Gauss-Bonnet forms, these include minimal area, Einstein-Hilbert, and, in higher than four dimensions, Lagrangians of quadratic and higher order. We find that, in immersion in frame bundles, they all lead to well set, causal and constraint-free theories. We speculate in Section 3 that this will be so generally.

We have recently discussed a well set and causal EDS for isometric immersion of (an orthonormal frame bundle over) Ricci-flat, vacuum spacetime in, as a target space, the 55 dimensional orthonormal frame bundle over flat (Euclidean or pseudo-Euclidean) ten dimensional space $E_{10}$ [20] [21] [22]. In that EDS (which was not derived from a Cartan form) there are four 3-forms, expressing both 
energy-momentum constraints and field equations. In Section 4 we introduce a closely related new variational EDS for a more general theory of gravitation, derived from a Cartan form that is just the Einstein-Hilbert Lagrangian, set on immersion of four dimensions in $E_{10}$. The resulting EDS has six 4-forms coding its field equations, but no 3 -form constraints. We calculate Cartan's characteristic integers and show that the EDS is well set and causal. The new EDS is contained in the former, so solutions of the former - Ricci-flat solutions - are a subset of the solutions of the more general theory. We show the Ricci-flat solutions to be selected when certain initial conditions are satisfied on an immersed three dimensional surface from which the final, causal, integration proceeds. In this final unique construction these conditions - the vanishing of the four 3-forms - are no longer required to be applied at each step as constraints.

The Einstein-Hilbert Lagrangian gives in fact well set generalized gravity theories on immersion of four dimensions in any higher dimensional frame bundle. The setting of initial conditions to specialize to Ricci-flatness, however, only works for immersion in $E_{10}$. This is of course the maximum immersion class of a general 4-geometry [24] [23]. Similarly treating $2+1$ gravity, generalized in this way, requires a (21 dimensional) frame bundle over six dimensional flat space $E_{6}$. This may be the natural first case to investigate for appropriateness of suggested constraint-free numerical integration schemes.

There may well be close connections of this work with known results in topological gravity and supergravity, which explore renormalizable gauge theories and lead on shell to the same extended Gauss-Bonnet Lagrangians that we use in immersion [25] [26]. We nevertheless should emphasize the differences, especially for dimension $p>3$. The gauge groups we deal with arise as intertwined structure equations for orthonormal frames and coframes, of dimension $p$ (even or odd) and $n-p$, respectively. They are not Poincare groups, but simply $O(p)$ and $O(n-p)$, up to signature. We have found reason to prefer $n=\frac{1}{2} p(p+1)$, the classic embedding dimension that does not a priori restrict the immersed metric [23] [24]. And we have not explored global implications of our results, much less the acceptability of quantum versions.

\section{Immersion geometries and the example of strings in four dimensions}

We will suppress the writing of the exterior multiplication operator throughout this note. Let $E_{n}$ be $n$ dimensional Euclidean or pseudo-Euclidean space. The 
structure equations for the basis forms of the orthonormal frame bundle over $E_{n}$ are

$$
\begin{array}{r}
d \omega^{\mu}+\omega_{\sigma}^{\mu} \omega^{\sigma}=0 \\
d \omega_{\nu}^{\mu}+\omega_{\sigma}^{\mu} \omega_{\nu}^{\sigma}=0,
\end{array}
$$

where $\mu, \nu=1, \ldots, n$. The $\omega_{\nu}^{\mu}$ are antisymmetric. The dimension of this space is $\frac{1}{2} n(n+1)$. Immersion of a $p$ dimensional subspace of $E_{n}$ is accomplished by (1) dividing the range of $\mu, \nu$ into two ranges, namely, $i, j=1, \ldots, p$ and $A, B=$ $p+1, \ldots, n$, and rewriting the structure equations 2.1 as

$$
\begin{array}{r}
d \omega^{i}+\omega_{k}^{i} \omega^{k}+\omega_{C}^{i} \omega^{C}=0 \\
d \omega^{A}+\omega_{k}^{A} \omega^{k}+\omega_{C}^{A} \omega^{C}=0 \\
d \omega_{j}^{i}+\omega_{k}^{i} \omega_{j}^{k}+\omega_{C}^{i} \omega_{j}^{C}=0 \\
d \omega_{A}^{i}+\omega_{k}^{i} \omega_{A}^{k}+\omega_{C}^{i} \omega_{A}^{C}=0 \\
d \omega_{B}^{A}+\omega_{k}^{A} \omega_{B}^{k}+\omega_{C}^{A} \omega_{B}^{C}=0,
\end{array}
$$

and then (2) forming the closed EDS generated by

$$
\left\{\omega^{A}, \omega_{i}^{A} \omega^{i}\right\}
$$

the vanishing of which selects subspaces which are so-called Darboux bundles.

The first $p+1$ Cartan characters of the EDS 2.3 are nonzero,

$$
s=\{n-p, \ldots, n-p, 0, \ldots, 0\},
$$

so from Cartan's theory the dimension $g$ of the subspace is calculated to be

$$
\frac{1}{2} p(p+1)+\frac{1}{2}(n-p)(n-p-1) .
$$

It has the structure of a fiber bundle over a $p$ dimensional base, since neither the $\omega_{j}^{i}\left(\frac{1}{2} p(p-1)\right.$ in number $)$ nor the $\omega_{B}^{A}\left(\frac{1}{2}(n-p)(n-p-1)\right.$ in number $)$ appear in the generators of the EDS. Corresponding to them are the Cartan characteristic vectors that appear as fibers in the solution subspaces. Clearly, they express the geometry of the orthonormal frames and coframes, induced at each point of a subspace. Pulled back (restricted) into the subspace the fields $\omega^{i}$ are in involution, i.e., at each point they remain independent and span every cross section. The $\omega_{j}^{i}$ 
constitute an orthonormal connection, and, from the third line of 2.2 , we see that the Riemann curvature 2-forms induced in the subspace are

$$
R_{j}^{i}=-2 \omega_{C}^{i} \omega_{j}^{C} .
$$

This explicit expression of the curvature in terms of basis forms whose structure equations are known is a key advantage of the immersion method. Given a Lagrangian involving $R_{j}^{i}$ it enables us to find an explicit EDS in terms of basis forms.

The last nonzero character of 2.3 is $s_{p}$, so this EDS is not causal: $s_{p}$ arbitrary functions of $p$ variables enter in the general solution. Additional dynamical equations must be added - in the guise of additional generating forms in the immersion EDS - to achieve $s_{p}=0$.

For the example of strings, with two dimensions immersed in four, $i, j=1,2$ and $A, B=3,4$, we have explicitly, in a 10 dimensional frame bundle over $E_{4}$, the generating forms of the EDS from 2.3:

$$
\begin{gathered}
\omega^{3} \\
\omega^{4} \\
\omega^{1} \omega_{1}^{3}+\omega^{2} \omega_{2}^{3} \\
\omega^{1} \omega_{1}^{4}+\omega^{2} \omega_{2}^{4} .
\end{gathered}
$$

These are to be annulled on any solution submanifold. We find $s=\{2,2,2,0\}$ and $g=4$. Solutions are $O(2) \otimes O(2)$ bundles over a 2 dimensional space, spanned by $\omega^{1}, \omega^{2}, \omega_{2}^{1}$, and $\omega_{4}^{3}$. A 2 dimensional cross section of a solution bundle gives an orthonormal framing of a metric 2-space, together with a realization of the connection $\omega_{2}^{1}$, the "second fundamental forms" $\omega_{i}^{3}$ and $\omega_{i}^{4}$, and also the field $\omega_{4}^{3}$ expressing arbitrary coframe orientation. With proper signs for signature the $E_{4}$ is Minkowski space and the immersed 2 dimensional space is a string.

Now consider the Lagrangian for extremal area, the Cartan form

$$
\Lambda=\omega^{1} \omega^{2} .
$$

Using 2.2, its exterior derivative is immediate:

$$
d \Lambda=\omega^{3}\left(\omega_{3}^{1} \omega^{2}-\omega_{3}^{2} \omega^{1}\right)+\omega^{4}\left(\omega_{4}^{1} \omega^{2}-\omega_{2}^{4} \omega^{1}\right)
$$

This result shows the "miracle" referred to by some authors [13]: a factor of each term is a form already in the immersion EDS, viz., $\omega^{3}$ and $\omega^{4}$. So if the other 
(2-form) factors in 2.7 are added to the EDS 2.5 , the result is that $d \Lambda$ is quadratic in the resulting EDS. This in turn means that, for an arbitrary vector field $V$ (in the bundle over $E_{4}$ ) the arbitrary variation of $\Lambda$, given by the Lie derivative

$$
L_{V}=V \cdot d \Lambda+d(V \bullet \Lambda),
$$

is contained in the resulting EDS and so vanishes when pulled back into a solution (modulo the exact or "boundary" term):

$$
V \bullet d \Lambda \subset \operatorname{EDS} \forall V .
$$

Thus this new EDS resulting from arbitrary variation of $\Lambda$ is generated by

$$
\begin{gathered}
\omega^{3} \\
\omega^{4} \\
\omega_{1}^{3} \omega^{1}+\omega_{2}^{3} \omega^{2} \\
\omega_{1}^{4} \omega^{1}+\omega_{2}^{4} \omega^{2} \\
\omega_{3}^{1} \omega^{2}-\omega_{3}^{2} \omega^{1} \\
\omega_{4}^{1} \omega^{2}-\omega_{2}^{4} \omega^{1} .
\end{gathered}
$$

This is the geometric understanding of a functional variational principle. For consistency, one must check closure, and calculate the Cartan characters to verify existence and uniqueness. In this case closure is immediate, and we find that $s=\{2,4,0,0\}$ and $g=4$. And now $s_{4}=0$. This extremal string theory is well set and causal; general solutions are determined by four functions set on an immersed line.

This process of construction of immersion EDS from postulated geometric Lagrangians is used in the following. The "miracle" occurs in all cases considered. But regardless of how they have been discovered the justification of the EDS is always the explicit demonstration of closure and Monte Carlo calculation of the causal Cartan characters [17].

\section{Lovelock Lagrangians for immersion in flat spaces}

We treat Lovelock Lagrangians [18] in $p$ dimension as $p$-forms that are exterior products of 1 -forms $\omega^{k}$ and 2-forms $R^{i j}$, completely antisymmetrized on indices. 
Also, $\omega^{k}$ must appear at least once, since in variation this generates the immersion forms $\omega^{A}$. These forms are known as dimensionally continued Gauss-Bonnet forms [25] [26]. We have discovered that, in immersion, using 2.2 and 2.4 , they are Cartan forms leading to well set and causal EDS.

For an immersed 2 dimensional space, $p=2$, as in our example in Section 2, the only Lovelock form is $\omega^{i} \omega^{j} \varepsilon_{i j}$.

For an immersed 3 dimensional space there are two Lovelock 3-forms: $\omega^{i} \omega^{j} \omega^{k} \varepsilon_{i j k}$ and $R^{i j} \omega^{k} \varepsilon_{i j k}$. To check the latter we calculate, using 2.2 (and remembering the range of $i, j=1,2,3)$,

$$
d\left(\omega_{i}^{A} \omega_{j}^{A} \omega^{k} \varepsilon_{i j k}\right)=-\omega^{B}\left(\omega_{B}^{k} \omega_{i}^{A} \omega_{k}^{j} \varepsilon_{i j k}\right) .
$$

This has the desired quadratic structure, i.e., it is a sum of terms each of which is the exterior product of an immersion 1-form and a dynamical 2-form. So we must check for closure, and for properties of the variational EDS generated by

$$
\left\{\omega^{B}, \omega_{i}^{B} \omega^{i}, \omega_{k}^{B} \omega_{i}^{A} \omega_{j}^{A} \varepsilon_{i j k}\right\} .
$$

Monte Carlo calculation of Cartan's characters indeed shows that this EDS is well set and causal for any number of immersing dimensions. In particular, for three dimensions immersed in six, $(A, B=4,5,6)$, which is the minimal immersion dimension for Ricci-flat (in this case, flat) 3 dimensional spaces, the orthonormal frame bundle dimension of 6 dimensional space is 21 and we calculate the Cartan characters to be $s=\{3,3,6,0,0,0,0,0,0\}$. Note that $s_{3}=0$. In the following we will not explicitly write any final zeros, so this would appear as $s=\{3,3,6\}$. Solutions are 9 dimensional bundles over 3 dimensional space with fibers allowing free rotation of triads in a connection $\left(\omega_{j}^{i}\right)$ and free rotation of the coframe at each point $\left(\omega_{B}^{A}\right)$.

For an immersed 4 dimensional space, the Lovelock 4-forms are $\omega^{i} \omega^{j} \omega^{k} \omega^{l} \varepsilon_{i j k l}$ and $R^{i j} \omega^{k} \omega^{l} \varepsilon_{i j k l}$ The first leads to a cosmological field. The second is the EinsteinHilbert Lagrangian. We discuss the theory which it leads to in Section 4. It turns out that initial conditions can be adjoined to select Ricci-flat solutions.

For an immersed 5 dimensional space, the Lovelock forms are $\omega^{i} \omega^{j} \omega^{k} \omega^{l} \omega^{m} \varepsilon_{i j k l m}$, $R^{i j} \omega^{k} \omega^{l} \omega^{m} \varepsilon_{i j k l m}$, and $R^{i j} R^{k l} \omega^{m} \varepsilon_{i j k l m}$ The new one leads to a quadratic gravity theory. Written out, this Lagrangian is

$$
\omega_{i}^{A} \omega_{j}^{A} \omega_{k}^{B} \omega_{l}^{B} \omega^{m} \varepsilon_{i j k l m}
$$

from which we derive the closed ideal

$$
\left\{\omega^{A}, \omega_{i}^{A} \omega^{i}, \omega_{i}^{A} \omega_{j}^{A} \omega_{k}^{B} \omega_{l}^{B} \omega_{m}^{C} \varepsilon_{i j k l m}\right\} .
$$


We calculated the characters of this, immersing 5 dimensions $(i, j=1, \cdots, 5)$ in $15(A, B=6, \cdots, 15)$ which has a 120 dimensional orthonormal frame bundle, to be

$$
\{10,10,10,10,20\}
$$

well set, causal, 60 dimensional solutions that are bundles over 5 , with 55 dimensional fibers expressing free rotation of frames and coframes $\omega_{j}^{i}$ and $\omega_{B}^{A}$. Five "initial condition" 4-forms can be adjoined to restrict solutions to those with a quadratic constraint on the Riemann tensor. Such restriction is explained in detail in Section 4 on Ricci-flat solutions.

In six dimensions the three Lovelock forms leading to well set immersion EDS are similar. In seven dimensions a cubic gravity theory appears. The pattern should now be evident. In all cases that we have calculated the variational EDS has been well set and causal.

\section{Constraint-free theories of gravity}

We now adopt the Einstein-Hilbert Lagrangian $R^{i j} \omega^{k} \omega^{l} \varepsilon_{i j k l}$ for immersion of 4 dimensional Riemannian geometry in a higher dimensional flat geometry, in particular in ten dimensions, $E_{10}$. Explicitly, this Lagrangian is

$$
\Lambda=\omega_{i}^{A} \omega_{j}^{A} \omega^{k} \omega^{l} \varepsilon_{i j k l} .
$$

Using 2.2 most terms in its exterior derivative cancel, leaving just

$$
d \Lambda=-2 \omega_{i}^{B} \omega_{j}^{B} \omega_{k}^{A} \omega^{l} \omega^{A} \varepsilon_{i j k l},
$$

which is quadratic in the immersion 1-forms $\omega^{A}$ and the six 4-forms $\omega_{i}^{B} \omega_{j}^{B} \omega_{k}^{A} \omega^{l} \varepsilon_{i j k l}$. So we consider the immersion EDS generated by

$$
\left\{\omega^{A}, \omega_{i}^{A} \omega^{i}, \omega_{i}^{B} \omega_{j}^{B} \omega_{k}^{A} \omega^{l} \varepsilon_{i j k l}\right\} .
$$

This system lives in the 55 dimensional orthonormal frame bundle over $E_{10}$. It may readily be checked for closure. Monte Carlo calculation of Cartan's characteristic integers yields $s=\{6,6,6,12\}$. Solutions are 25 dimensional, with 21 dimensional fibers over a 4 dimensional base.

Note in particular we have found $s_{3}=12$ and $s_{4}=0$, so, in Cartan's nested construction of solutions [17], after vectors $V_{1}, V_{2}$, and $V_{3}$ are found, spanning an immersed 3 dimensional space, 12 initial value functions on it determine the 
final vector field $V_{4}$ by quadrature, causally and essentially uniquely, i.e., up to choice of arbitrary admixture of the 21 Cartan characteristic vectors. Vectors $V_{1}$, $V_{2}$, and $V_{3}$ are dragged along $V_{4}$ as it is constructed. The $\omega^{i}$ are involutory, so linear combinations of the $V_{i}(i=1, \cdots, 4)$, may be taken at each point so that $V_{i} \bullet \omega^{j}=\delta_{i}^{j}$ everywhere. The Riemann tensor components induced in a solution with respect to this orthonormal frame are

$$
R_{i j k l}=2 V_{i} \bullet V_{j} \bullet \omega_{k}^{A} \omega_{l}^{A}
$$

and the Einstein tensor components are

$$
E_{l p}=-\frac{1}{6} V_{i} \bullet V_{j} \bullet V_{k} \bullet \omega_{r}^{A} \omega_{s}^{A} \omega^{t} \varepsilon_{i j k l} \varepsilon_{r s t p}
$$

If entirely intrinsic tetrad expressions for the theory are desired, six symmetric second fundamental forms, denoted by $K_{i j}^{A}$, may be introduced to describe the immersion at each point via

$$
\omega_{i}^{A}=K_{i j}^{A} \omega^{j}
$$

and the inner products 4.2 and 4.3 may be expanded. The Einstein tensor becomes

$$
E_{p q}=K_{p s}^{A} K_{s q}^{A}-K_{s s}^{A} K_{p q}^{A}+\frac{1}{2}\left(K_{s s}^{A} K_{t t}^{A}-K_{s t}^{A} K_{s t}^{A}\right) \eta_{p q}
$$

In the case of positive definite signature we have $\eta_{p q}=\delta_{p q}$. Otherwise, of course, the correct signature for $\eta_{p q}$ is used and the contractions in 4.5 are to reflect the correct signature.

The dynamical conditions, coded by the $4-$ forms in 4.1 , are third order in the $K_{i j}^{A}$. They lead to

$$
K_{s t}^{A} E^{s t}=0 .
$$

Explicit differential equations for the generalized theory, with $K_{i j}^{A}$ as dependent variables, are obtained on substitution of 4.4 into the structure equations 2.2 , modulo 4.6. As with all tetrad (or spinor) formulations we get not exactly partial differential equations, since the independent variables are implicit in the $\omega^{i}$. Rather, such sets of equations are derivational, i.e., first order in directional derivatives along the $V_{i}$. Second order partial differential equations result from introducing coordinates and metric fields $g_{i j}$.

Now, as explained in the Introduction, our previously discussed EDS for Ricciflat 4 dimensional spaces immersed in $E_{10}$ was generated by immersion forms and the Einstein 3-forms $\omega_{i}^{A} \omega_{j}^{A} \omega^{k} \varepsilon_{i j k l}$ [20]. The EDS of the more general theory, 4.1, is contained in the former, so Ricci-flat solutions, i.e., $E_{l p}=0$, are special cases. 
This is also clear from 4.6. Both theories are causal because $s_{4}=0$. The vector $V_{4}$ is unique. If $V_{1}, V_{2}$, and $V_{3}$, which span a 3 dimensional surface $\Sigma$, satisfy already the conditions for Ricci-flatness, i.e., they annul the 3-forms at every point of $\Sigma$, the final unique quadrature for $V_{4}$ (using the 4 -forms only) will, without further constraint, construct a Ricci-flat solution. We have verified this by calculating the Cartan characters, and explicit components of $V_{1}, V_{2}$, and $V_{3}$ using equations annulling the four 3 -forms, whereas for $V_{4}$ only using the $4-$ forms in 4.1 . The result was $s=\{6,6,10,8\}$, and the four vectors, taken three at a time, annulled all 10 components of the four 3 -forms $E_{l p}$.

The specialization to Ricci-flat solutions by imposition only of initial conditions (without further 3-form constraints) may also be understood intrinsically. We have six homogeneous linear equations for the ten $E_{i j}$ in 4.6 , and we impose the four conditions $E_{i 4}=0$ on $\Sigma$. Thus all $E_{p l}=0$ on $\Sigma$. It is well known that the components $E_{i 4}$ then remain zero in a neighborhood of $\Sigma$. This follows from the contracted Bianchi identities, which express energy-momentum conservation, in Einstein's interpretation of $E_{i j}$. So all ten $E_{p l}$ are zero in the neighborhood and a Ricci-flat solution has been selected, just by imposition of initial conditions. 


\section{References}

[1] Goldschmidt $\mathrm{H}$ and Sternberg S 1973 The Hamilton-Cartan formalism in the calculus of variations Ann. Inst. Fourier, Grenoble 23, 1 203-67. Uses modern differential geometric notation, and contains references to Weyl, Caratheodory, de Donder and Lepage.

[2] Nono T and Mimura F 1972 A differential geometric formalism for multiple integral variational problems, Tensor, N.S. 26, 79-85

[3] Mimura F 1974 Generalized formalism of mechanics, Bull. Kyushu. Inst. Tech. 21

[4] Dedecker P 1977 On the generalization of symplectic geometry to multiple integrals in the calculus of variations, in Bleuler $\mathrm{K}$ and Reetz A, eds. Lecture Notes in Mathematics \#570 (Berlin: Springer)

[5] Hermann R 1977 Differential Geometry and the Calculus of Variations, 2nd edition, chapter 37 (Brookline, MA: Math Sci Press)

[6] Kijowski J and Tulczyjew WM 1979 A symplectic framework for field theories, in Lecture Notes in Physics \#107 (Springer).

[7] Estabrook FB 1980 Differential geometry as a tool for applied mathematics, in Martini R, ed. Lecture Notes in Mathematics \#810 (Berlin: Springer)

[8] Francaviglia M and Krupka D 1982 Ann. Inst. H. Poincare 37295

[9] Betounes DE 1984 Extension of the classical Cartan form Phys. Rev. D 29 599-606. Also 1984 Jour. Math. Phys. 28 2347-53

[10] Bauderon M 1985 Differential geometry and Lagrangian formalism in the calculus of variation, in Rassias GM and Rassias TM, eds. Differential Geometry, Calculus of Variations and their Applications, Lecture Notes in Pure and Applied Mathematics \#100 67-82 (New York: Marcel Dekker)

[11] Burke WL 1985 Applied Differential Geometry (Cambridge: Cambridge University Press) 
[12] Rund H A 1985 A Cartan from for the field theory of Caratheodory in the calculus of variations of multiple integrals, in Rassias GM and Rassias TM, eds. Differential Geometry, Calculus of Variations and their Applications, Lecture Notes in Pure and Applied Mathematics \#100 455-469 (Marcel Dekker: New York and Basel).

[13] Hermann R 1988 Differential from methods in the theory of variational systems and Lagrangian field theories Acta Appl. Mathematicae 12 35-78. Treats minimal submanifolds.

[14] Gotay MJ 1991 An exterior differential system approach to the Cartan form, in Symplectic geometry and mathematical physics, Proc. colloquium in honor of J-M Souriau, Aix-en-Provence 1990, eds. Donato P, Elhadad J and Tuynmen GM, Prog. Math 99 (Boston: Birkhauser)

[15] Yang K 1992 Exterior Differential Systems and Equivalence Problems (Amsterdam: Kluwer)

[16] Bryant R, Chern S-S, Gardner R, Goldschmidt H and Griffiths P 1991 Exterior Differential Systems (New York: Springer-Verlag)

[17] Estabrook FB and Wahlquist HD 1989 Classical geometries defined by exterior differential systems on higher frame bundles Class. Quantum Grav. 6 263-274

[18] Rund H 1971 Invariant theory of variational problems on subspaces of a Riemannian manifold, Hamburger Mathematische Einzelschriften Neue Folge Heft 5 (Gottingen: Vandenhoeck \& Ruprecht)

[19] Lovelock D 1971 The Einstein Tensor and Its Generalizations J. Math. Phys. 12 498-501

[20] Estabrook FB and Wahlquist HD 1993 Immersion ideals and the causal structure of Ricci-flat geometries Class. Quantum Grav. 10 1851-58

[21] Estabrook FB 1991 Lagrangians for Ricci-flat geometries Class. Quantum Grav. 8 L151-L154

[22] Estabrook FB, Robinson RS and Wahlquist HD 1997 Hyperbolic equations for vacuum gravity using special orthonormal frames Class. Quantum Grav. 14 1237-1247 
[23] Eisenhart LP 1949 Riemannian Geometry (Princeton: Princeton University Press) See page 143.

[24] Kramer D, Stephani H, MacCallum M and Herlt E 1980 Exact Solutions of Einstein's Field Equations (Cambridge: Cambridge University Press) See Chapter 32.

[25] Chamseddine AH 1990 Topological gravity and supergravity in various dimensions Nucl. Phys. B 346 213-234

[26] Muller-Hoissen F 1990 From Chern-Simons to Gauss-Bonnet Nucl. Phys. B $346235-252$ 